\title{
A Study on Effective Study Path for Open Education Students Based on Complex Network
}

\author{
He Junying ${ }^{a}$, Yang Binhua ${ }^{b}$ \\ School of Economics and Management, Yunnan Open University, Yunnan, China \\ ahejunying_edu@163.com, byangbinghua@ynou.edu.cn
}

Keywords: complex network, knowledge relation network, effective study path

\begin{abstract}
This paper is based on the constructivism learning theory. The knowledge nodes in study were taken as network nodes to build complex knowledge nodes relation network model by applying the method to build a complex network, to find the basic effective study paths for open education students. In building the above-mentioned model, core points including importance of knowledge node, relevance and path selection and etc. were discussed.
\end{abstract}

\section{Introduction}

According to the constructivism learning theory, study is a process for a student to build his/her own knowledge. Study is not a process to passively receive information stimulation but to actively build recognition and process and treat external information based on the experiences of student to make sense of information ${ }^{[1]}$. In open education, students actively conduct self-directed learning by using text books, lecture slides and plenty of other digitized resources. In self-directed learning, students flexibly complete a series of meaning construction activities including selecting resources for study, reading and thinking. The resources selection and processing involved in the study process of students are recorded by using a timeline and is called study path. Discovering the effective learning path for subsequently organizing learning resources effectively for studying is a hotspot in Open Education Research.

In open education, learning resources and knowledge nodes are not randomly selected for students but are based on relevant theory framework and relations among knowledge nodes in the knowledge network of learning contents, and students can actively select knowledge sequence with meaning construction for studying ${ }^{[2]}$. The knowledge sequence is the effective learning path. It is significantly beneficial for the study of the effective learning path to apply the method of building a complex network and make use of knowledge nodes in study as network nodes to build complex knowledge relation network model.

\section{Complex Knowledge Relation Network Model}

\subsection{Complex Network}

Complex network is not strictly defined, and it can be deemed as a topology abstracted from 
plenty of complex systems. It is neither a regular network nor a random network. Existing networks in real society have confirmed and random characteristics that are hidden in statistical characteristics. Therefore, it is very important to attain the statistic description of all characteristics of complex networks ${ }^{[3]}$.

Common statistical properties include degree distribution, average path length and clustering coefficient. Degree basically describes the statistics characteristics of connection among nodes, and it also reflects key network evolutionary characters. The average path length of network represents the nodes experienced by matter, information and energy going from one node to other nodes in a network. The fewer nodes they met, the higher the transmission efficiency will be. The clustering coefficient and distribution of network can reflect the density of nodes in a network, and it shows the connectedness of nodes in a network.

\subsection{Knowledge Relation Network Model}

The overall concept design to build a proper knowledge relation network model is: first, breaking down a course content into relatively independent knowledge nodes that are musts for active meaning building for students; Secondly, using these knowledge nodes as network nodes in the relation network model and analyzing the importance of each knowledge node in the overall network model; at last, analyzing the relation among knowledge nodes and defining the relevancy amongst network nodes. Then the base for analyzing and studying effective learning path for students is prepared.

During the study, the learning path of effective students in open education was analyzed, and it is found that there are basic effective learning paths for different course contents in active meaning building.

- For common course contents, students complete the learning of all knowledge nodes (P denotes a knowledge node) linearly. The topology in the knowledge relation network is shown in Figure 1.

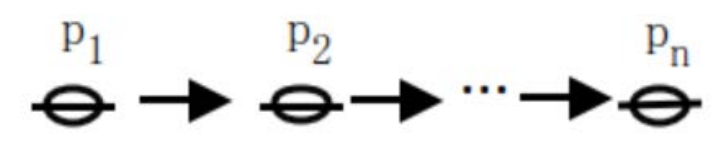

Figure 1 Topology 1 in Knowledge Relation Network

- For difficult and key course contents, students tends to choose to study knowledge nodes that are related to the current knowledge nodes before study knowledge nodes that are irrelevant to the current knowledge nodes. The topology in the knowledge relation network is shown in Figure 2.

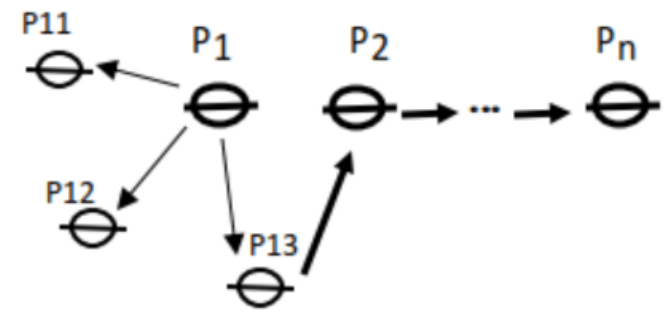

Figure 2 Topology 2 in Knowledge Relation Network

- For complex knowledge nodes, students study relevant knowledge nodes linearly before studying irrelevant knowledge nodes; during the process, they constantly review core knowledge 
nodes. The topology in the knowledge relation network is as shown in Figure $3 . \mathrm{P}_{2}$ denotes a core knowledge node. In the effective learning path, students need to review $\mathrm{P}_{2}$ and $\mathrm{P}_{23}$ knowledge nodes in the learning process.

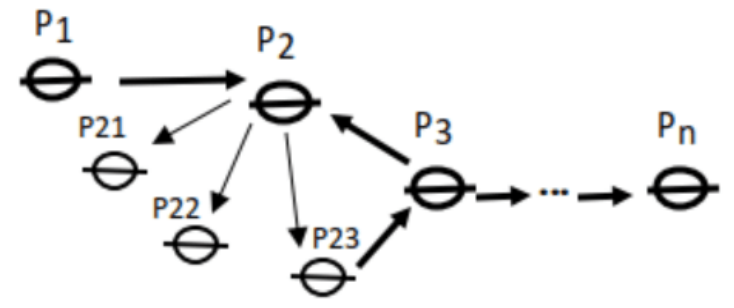

Figure 3 Topology 3 in Knowledge Relation Network

\section{Process to Build Complex Knowledge Relation Network}

According to the idea to build the knowledge relation network, the following three key problems need to be solved in building the complex knowledge relation network.

First, the importance of a knowledge node in a course. A course includes a lot of knowledge nodes i.e. average, common, difficult and key knowledge nodes. Therefore, the priority in building complex knowledge relation network is to determine the importance of a specific knowledge node in a course.

Secondly, the relation amongst knowledge nodes A knowledge node can be related to another knowledge node or nodes in one way or the other in a course, which significantly influence the sequence of knowledge nodes selection and the learning path. Thus, it is of vital significance to quantify the relation amongst knowledge nodes in building complex knowledge relation network.

At last, exploring the effective learning path in learning knowledge nodes. The objective of building complex knowledge relation network is to unearth the effective learning path in learning knowledge nodes.

\subsection{Importance of Specific Knowledge Node}

The importance of a specific knowledge node is mainly determined by the frequency of occurrence of relevant terminology related to a knowledge node, and it is analyzed, quantified and denoted by using TF-IDF ${ }^{[4]}$ word frequency weighting technique. The importance of knowledge node i (Wi) can be expressed with the equation.

$$
W_{i}=\sum_{1}^{n} W_{k}
$$

In the equation, $\mathrm{K}$ denotes relevant terminology related to the knowledge node $\mathrm{i}, \mathrm{W}_{\mathrm{k}}$ denotes the importance of terminology $\mathrm{K}$ in a course, and it can be expressed in the equation.

$$
W_{k}=f_{k} \times \log _{d} N
$$

In the equation, $\mathrm{f}_{\mathrm{K}}$ denotes the frequency of occurrence of terminology $\mathrm{K}$ in the course, $\mathrm{N}$ denotes the all knowledge nodes in a course, d denotes the frequency of occurrence of $\mathrm{K}$ in all knowledge nodes. 


\subsection{Relation amongst Knowledge Nodes}

The relation amongst knowledge nodes can be denoted with a Cosine similar function ${ }^{[5]}$. Assuming that knowledge node $\mathrm{A}$ and knowledge node $\mathrm{B}$ shares $\mathrm{m}$ terminologies, the relation between $\mathrm{A}$ and $\mathrm{B}\left(R_{A B}\right)$ can be expressed with the equation.

$$
R_{A B}=\frac{\sum_{1}^{m} W_{A m} \times W_{B m}}{\sqrt{\sum_{1}^{m} W_{A m}{ }^{2} \times \sqrt{\sum_{1}^{m} W_{B m}{ }^{2}}}}
$$

In the equation, $W_{A m}$ denotes the importance of $\mathrm{m}$ in knowledge node $\mathrm{A}$, and $W_{B m}$ denotes the importance of $\mathrm{m}$ in knowledge node $\mathrm{B}$. $R_{A B}$ stays within [-1, 1]. If $R_{A B}$ is close to 1 , the relation between $\mathrm{A}$ and $\mathrm{B}$ is high, otherwise, it is low.

$$
R=\left[\begin{array}{ccc}
R_{11} & \cdots & R_{1 n} \\
\vdots & \ddots & \vdots \\
R_{n 1} & \cdots & R_{n n}
\end{array}\right]
$$

The relation amongst knowledge nodes for a course can be expressed as a multi-dimensional spatial vector. If a course has $n$ knowledge nodes, the following matrix can be used to quantify and save the relation amongst knowledge nodes.

\subsection{Effective Study Path}

An effective learning path must meet three requirements: all the knowledge nodes are reachable; any core knowledge node of a vital knowledge network can be passed many times; and strongly related knowledge nodes are the first choice to pass.

The distance between any two knowledge nodes $\mathrm{i}$ and $\mathrm{j}$ in a complex knowledge network is defined as the learning path. Dij denotes the number of connection segments of the shortest path between $\mathrm{i}$ and $\mathrm{j}$. The average shortest path length $\mathrm{L}$ for a network equals to the average of shortest path of any two nodes in a knowledge network. For a knowledge network, the shorter the average path length is, the higher the transmission efficiency of material, energy and information transmission will be. Therefore, the effective learning path $\mathrm{L}$ can be expressed as the equation.

$$
L=\frac{1}{N(N-1)} \times \sum_{i \neq j} D_{i j}
$$

In the equation, $\mathrm{N}$ denotes the number of knowledge nodes for a course. It must be ensured that effective learning path $\mathrm{L}$ can reach all knowledge nodes and reach knowledge nodes with strong relation first, and core knowledge nodes are repeatedly used in multiple learning paths.

\subsection{Process to Extract Effective Study Path}

The different attributes of knowledge node Pi can be expressed with a five tuple (ID, SUB, COR, CON, WEI) .ID denotes the identification of knowledge node; SUB denotes the subject of knowledge node; COR denotes the course of knowledge node; CON denotes the content of knowledge node; WEI denotes the weight of knowledge node.

The similarity between knowledge nodes can be expressed by $R_{A B}(P i, P j)$. When $R_{A B}(P i, P j)=1$, $\mathrm{Pi}$ and $\mathrm{Pj}$ are necessarily experienced nodes in the learning process from $\mathrm{A}$ subject to $\mathrm{B}$ subject, knowledge nodes $\mathrm{Pi}$ and $\mathrm{Pj}$ is reachable. The following matrix can be used to quantify the 
reachability between any two knowledge nodes.

$$
A i j=\left\{\begin{array}{c}
1, \quad R i j=1 \\
0, R i j=0,
\end{array}(i, j=1,2 \ldots n)\right.
$$

The learning path from the A subject to the B subject is the sum of the reachable paths of all the necessarily experienced knowledge nodes, such that there are many learning paths. The effective learning path is the minimum of the total reachable path.

\section{Conclusion}

This paper proposed the idea to build the knowledge relation network model based on the complex network theory, and studied the effective learning path for open education. In designing curriculum for open education, it can be used to effectively organize resources and carry out learning activities. It provides a foundation for students to actively build their own knowledge system, which improves efficiency and outcome of learning.

\section{Acknowledgment}

This research is supported by 2016 Application Basic Research Program of Yunnan Province "Research on Learning Support Behavior in Open Education based on Multi-agent"(ID: 2016FD068)

\section{References}

[1] Yunfang Yang. A summary of Constructivist Learning Theory [J]. Forum on Contemporary Education, 2005, (8): 35-36

[2] Lianglian Cao. Learning path analysis and learning behavior characteristics in online learning [J]. Distance Education in China, 2014, (4): 25-30

[3] Nan, He, Yide, Li, Wenyan, Gan, Xi, Zhu. Mining Vital Nodes in Complex Networks [J]. Computer Science, 2007, (12): $1-5$

[4] Kewen Chen, Zuping Zhang, Jun Long, Hao Zhang. Turning from TF-IDF to TF-IGM for term weighting in text classification [J]. Expert Systems With Applications, 2016, 66: 245-260

[5] Yangyi Dong, Weihua Li, HuiYu. Hierarchical relation mining of Chinese text based on mixed cosine similarity [J]. Application Research of Computers, 2017, 34(5): 1406-1409 